\title{
Vertex PI Index and Szeged Index of Certain Special Molecular Graphs
}

\author{
Li Yan ${ }^{1}$, Junsheng $\mathrm{Li}^{1 *}$, Wei Gao ${ }^{2}$ \\ ${ }^{1}$ School of Engineer, Honghe University, Mengzi 661100, China \\ ${ }^{2}$ School of Information Science and Technology, Yunnan Normal University, Kunming 650500, China
}

\begin{abstract}
The vertex PI index and Szeged index are distance-based topological index which reflect certain structural features of organic molecules. Each structural feature of such organic molecule can be expressed as a graph. In this paper, we determine the vertex PI index and Szeged index of fan molecular graph, wheel molecular graph, gear fan molecular graph, gear wheel molecular graph, and their r-corona molecular graphs.
\end{abstract}

Keywords: Chemical graph theory, vertex PI index, Szeged index, Fan molecular graph, Wheel molecular graph, Gear fan molecular graph, Gear wheel molecular graph, r-corona molecular graph.

\section{INTRODUCTION}

Wiener index, PI index, Hyper-wiener index, Geometricarithmetic index and Zagreb indices are introduced to reflect certain structural features of organic molecules. Several papers contributed to determine the distance-based index of special molecular graphs (See Yan et al., [1, 2], Gao and Shi [3] for more detail). Let $P_{n}$ and $C_{n}$ be path and cycle with $n$ vertices. The molecular graph $F_{n}=\{v\} \vee P_{n}$ is called a fan molecular graph and the molecular graph $W_{n}=\{v\} \vee C_{n}$ is called a wheel molecular graph. Molecular graph $I_{r}(G)$ is called $r$-crown molecular graph of $G$ which splicing $r$ hang edges for every vertex in $G$. By adding one vertex in every two adjacent vertices of the fan path $P_{n}$ of fan molecular graph $F_{n}$, the resulting molecular graph is a subdivision molecular graph called gear fan molecular graph, denote as $\tilde{F}_{n}$. By adding one vertex in every two adjacent vertices of the wheel cycle $C_{n}$ of wheel molecular graph $W_{n}$, The resulting molecular graph is a subdivision molecular graph, called gear wheel molecular graph, denoted as $\tilde{W}_{n}$.

Let $e=u v$ be an edge of the molecular graph $G$. The number of vertices of $G$ whose distance to the vertex $u$ is smaller than the distance to the vertex $v$ is denoted by $n_{u}(e)$. Analogously, $n_{v}(e)$ is the number of vertices of $G$ whose distance to the vertex $v$ is smaller than the distance to the vertex $u$. Note that vertices equidistant to $u$ and $v$ are not counted. The vertex PI index of $G$ is defined as

$$
P I_{v}(G)=\sum_{e=u v}\left[n_{u}(e)+n_{v}(e)\right] .
$$

Khalifeh et al., [4] presented the vertex PI indices of cartesian product molecular graphs. Ashrafi et al., [5]

*Address correspondence to these authors at the School of Engineer, Honghe University, Mengzi 661100, China; Tel: +86 139-8733-0109;

E-mails: yanli@uoh.edu.cn; 904382815@qq.com studied the vertex PI index of an infinite family of fullerenes. Khalifeh et al., [6] raised a matrix method for computing vertex PI index of molecular graphs. Yousefi-Azari [7] calculated vertex-PI index of single and multi-walled nanotubes. Mansour and Schork [8] determined the vertex PI index of bridge molecular graphs. Nadjafi-Arani et al., [9] presented the extremal molecular graphs with respect to the vertex PI index. Das and Gutman [10] obtained the bound for vertex PI index by virtue of simple molecular graph parameters. Li et al., [11] computed the vertex PI of chain molecular graphs. Bahrami and Yazdani [12] yielded the vertex PI index of $V$-phenylenic nanotubes and nanotori.

The Szeged index is closely related to the Wiener index and defined as

$S z(G)=\sum_{e=u v} n_{u}(e) n_{v}(e)$.

Some conclusion for Szeged index can refer to [13].

In this paper, we present the vertex PI index and Szeged index of $I_{r}\left(F_{n}\right), I_{r}\left(W_{n}\right)$, and $I_{r}\left(\tilde{W}_{n}\right)$.

\section{VERTEX PI INDEX}

\section{Theorem 1:}

$$
P I_{v}\left(I_{r}\left(F_{n}\right)\right)=r^{2}\left(n^{2}+2 n+1\right)+r\left(2 n^{2}+5 n-3\right)+\left(n^{2}+3 n-4\right) \text {. }
$$

Proof. Let $P_{n}=v_{1} v_{2} \ldots v_{n}$ and the $r$ hanging vertices of $v_{i}$ be $v_{i}^{1}$ $, v_{i}^{2}, \ldots, v_{i}^{r}(1 \leq i \leq n)$. Let $v$ be a vertex in $F_{n}$ beside $P_{n}$, and the $r$ hanging vertices of $v$ be $v^{1}, v^{2}, \ldots, v^{r}$. Using the definition of vertex PI index, we have

$$
P I_{v}\left(I_{r}\left(F_{n}\right)\right)=\sum_{i=1}^{r}\left(n_{v}\left(v v^{i}\right)+n_{v^{i}}\left(v v^{i}\right)\right)+
$$




$$
\begin{aligned}
& \sum_{i=1}^{n}\left(n_{v}\left(v v_{i}\right)+n_{v_{i}}\left(v v_{i}\right)\right)+\sum_{i=1}^{n-1}\left(n_{v_{i}}\left(v_{i} v_{i+1}\right)+n_{v_{i+1}}\left(v_{i} v_{i+1}\right)\right) \\
& \sum_{i=1}^{n} \sum_{j=1}^{r}\left(n_{v_{i}}\left(v_{i} v_{i}^{j}\right)+n_{v_{i}^{j}}\left(v_{i} v_{i}^{j}\right)\right) \\
& =r(2 n(r+1))+(2 n(r+1)+(n-2)(n-1)(r+1))+2(3 r+3)+ \\
& (n-3)(4 r+4)+n r(n+1)(r+1) \\
& =r^{2}\left(n^{2}+2 n+1\right)+r\left(2 n^{2}+5 n-3\right)+\left(n^{2}+3 n-4\right) .
\end{aligned}
$$

Corollary 1. $P I_{v}\left(F_{n}\right)=n^{2}+3 n-4$.

Theorem 2.

$P I_{v}\left(I_{r}\left(W_{n}\right)\right)=r^{2}\left(n^{2}+2 n+1\right)+r\left(2 n^{2}+5 n+1\right)+\left(n^{2}+3 n\right)$.

Proof. Let $C_{n}=v_{1} v_{2} \ldots v_{n}$ and $v_{i}^{1}, v_{i}^{2}, \ldots, v_{i}^{r}$ be the $r$ hanging vertices of $v_{i}(1 \leq i \leq n)$. Let $v$ be a vertex in $W_{n}$ beside $C_{n}$, and $v^{1}, v^{2}, \ldots, v^{r}$ be the $r$ hanging vertices of $v$. We denote $v_{n} v_{n+1}=v_{n} v_{1}$. In view of the definition of vertex PI index, we infer

$$
\begin{aligned}
& P I_{v}\left(I_{r}\left(W_{n}\right)\right)=\sum_{i=1}^{r}\left(n_{v}\left(v v^{i}\right)+n_{v^{i}}\left(v v^{i}\right)\right)+ \\
& \sum_{i=1}^{n}\left(n_{v}\left(v v_{i}\right)+n_{v_{i}}\left(v v_{i}\right)\right)+\sum_{i=1}^{n}\left(n_{v_{i}}\left(v_{i} v_{i+1}\right)+n_{v_{i+1}}\left(v_{i} v_{i+1}\right)\right) \\
& \sum_{i=1}^{n} \sum_{j=1}^{r}\left(n_{v_{i}}\left(v_{i} v_{i}^{j}\right)+n_{v_{i}^{j}}\left(v_{i} v_{i}^{j}\right)\right) \\
& =r(n+1)(r+1)+n(n-1)(r+1)+n(4 r+4)+n r(n+1)(r+1) \\
& =r^{2}\left(n^{2}+2 n+1\right)+r\left(2 n^{2}+5 n+1\right)+\left(n^{2}+3 n\right) .
\end{aligned}
$$

Corollary 2. $P I_{v}\left(W_{n}\right)=n^{2}+3 n$.

Theorem 3. $P I_{v}\left(I_{r}\left(\tilde{F}_{n}\right)\right)=4 n^{2} r^{2}+r\left(10 n^{2}-4 n\right)+\left(6 n^{2}-4 n\right)$.

Proof. Let $P_{n}=v_{1} v_{2} \ldots v_{n}$ and $v_{i, i+1}$ be the adding vertex between $v_{i}$ and $v_{i+1}$. Let $v_{i}^{1}, v_{i}^{2}, \ldots, v_{i}^{r}$ be the $r$ hanging vertices of $v i(1 \leq i \leq n)$. Let $v_{i, i+1}^{1}, v_{i, i+1}^{2}, \ldots, v_{i, i+1}^{r}$ be the $r$ hanging vertices of $v_{i, i+1}(1 \leq i \leq n-1)$. Let $v$ be a vertex in $F_{n}$ beside $P_{n}$, and the $r$ hanging vertices of $v$ be $v^{1}, v^{2}, \ldots, v^{r}$.

By virtue of the definition of vertex PI index, we yield

$$
\begin{aligned}
& P I_{v}\left(I_{r}\left(\tilde{F}_{n}\right)\right)=\sum_{i=1}^{r}\left(n_{v}\left(v v^{i}\right)+n_{v^{i}}\left(v v^{i}\right)\right)+\sum_{i=1}^{n}\left(n_{v}\left(v v_{i}\right)+n_{v_{i}}\left(v v_{i}\right)\right) \\
& +\sum_{i=1}^{n} \sum_{j=1}^{r}\left(n_{v_{i}}\left(v_{i} v_{i}^{j}\right)+n_{v_{i}^{j}}\left(v_{i} v_{i}^{j}\right)\right)+\sum_{i=1}^{n-1}\left(n_{v_{i}}\left(v_{i} v_{i, i+1}\right)+n_{v_{i, i+1}}\left(v_{i} v_{i, i+1}\right)\right)+ \\
& \sum_{i=1}^{n-1}\left(n_{v_{i, i+1}}\left(v_{i, i+1} v_{i+1}\right)+n_{v_{i+1}}\left(v_{i, i+1} v_{i+1}\right)\right)
\end{aligned}
$$

$$
\begin{aligned}
& \sum_{i=1}^{n-1} \sum_{j=1}^{r}\left(n_{v_{i, i+1}}\left(v_{i, i+1} v_{i, i+1}^{j}\right)+n_{v_{i, i+1}^{j}}\left(v_{i, i+1} v_{i, i+1}^{j}\right)\right) \\
= & r(2 n(r+1))_{+} 2 \times 2 n(r+1)+. .+n r \times 2 n(r+1)+ \\
& (n-1) 2 n(r+1)_{+}(n-1) 2 n(r+1)_{+}(n-1) r \times 2 n(r+1) \\
= & 4 n^{2} r^{2}+r\left(10 n^{2}-4 n\right)+\left(6 n^{2}-4 n\right) .
\end{aligned}
$$

Corollary 3. $P I_{v}\left(\tilde{F}_{n}\right)=6 n^{2}-4 n$.

\section{Theorem 4.}

$$
\begin{aligned}
& P I_{v}\left(I_{r}\left(\tilde{W}_{n}\right)\right)= \\
& r^{2}\left(4 n^{2}+4 n+1\right)+r\left(10 n^{2}+7 n+1\right)+\left(6 n^{2}+3 n\right) .
\end{aligned}
$$

Proof. Let $C_{n}=v_{1} v_{2} \ldots v_{n}$ and $v$ be a vertex in $W_{n}$ beside $C_{n}$, and $v_{i, i+1} \square$ be the adding vertex between $v_{i}$ and $v_{i+1}$. Let $v^{1}$, $v^{2}, \ldots, v^{r}$. be the $r$ hanging vertices of $v$ and $v_{i}^{1}, v_{i}^{2}, \ldots, v_{i}^{r}$ be the $r$ hanging vertices of $v_{i}(1 \leq i \leq n)$. Let $v_{n, n+1}=v_{1, n}$ and $v_{i, i+1}^{1}, v_{i, i+1}^{2}, \ldots, v_{i, i+1}^{r}$ be the $r$ hanging vertices of $v_{i, i+1}$ $(1 \leq i \leq n)$. Let $v_{n . n+1}=v_{n .1}, \quad v_{n+1}=v_{1}$. In view of the definition of vertex PI index, we deduce

$$
\begin{aligned}
& P_{v}\left(I_{r}\left(\tilde{W}_{n}\right)\right)=\sum_{i=1}^{r}\left(n_{v}\left(v v^{i}\right)+n_{v^{i}}\left(v v^{i}\right)\right)+ \\
& \sum_{i=1}^{n}\left(n_{v}\left(v v_{i}\right)+n_{v_{i}}\left(v v_{i}\right)\right) \\
& +\sum_{i=1}^{n} \sum_{j=1}^{r}\left(n_{v_{i}}\left(v_{i} v_{i}^{j}\right)+n_{v_{i}^{j}}\left(v_{i} v_{i}^{j}\right)\right)+ \\
& \sum_{i=1}^{n}\left(n_{v_{i}}\left(v_{i} v_{i, i+1}\right)+n_{v_{i, i+1}}\left(v_{i} v_{i, i+1}\right)\right) \\
& +\sum_{i=1}^{n}\left(n_{v_{i, i+1}}\left(v_{i, i+1} v_{i+1}\right)+n_{v_{i+1}}\left(v_{i, i+1} v_{i+1}\right)\right) \\
& +\sum_{i=1}^{n} \sum_{j=1}^{r}\left(n_{v_{i, i+1}}\left(v_{i, i+1} v_{i, i+1}^{j}\right)+n_{v_{i, i+1}^{j}}\left(v_{i, i+1} v_{i, i+1}^{j}\right)\right)= \\
& r(2 n+1)(r+1){ }_{+} n(2 n+1)(r+1)+n r(2 n+1)(r+1)+ \\
& n(2 n+1)(r+1)+n(2 n+1)(r+1)+n r(2 n+1)(r+1) \\
& =r^{2}\left(4 n^{2}+4 n+1\right)+r\left(10 n^{2}+7 n+1\right)+\left(6 n^{2}+3 n\right) .
\end{aligned}
$$

Corollary 4. $P I_{v}\left(\tilde{W}_{n}\right)=6 n^{2}+3 n$.

\section{SZEGED INDEX}

The notations for certain special molecular graphs can refer to Theorem 1- Theorem 4.

\section{Theorem 5.}

$S z\left(I_{r}\left(F_{n}\right)\right)=r^{2}\left(2 n^{2}+4 n-5\right)+r\left(3 n^{2}+5 n-12\right)+\left(n^{2}+2 n-6\right)$. 
Proof. Using the definition of Szeged index, we have

$$
\begin{aligned}
& S z\left(I_{r}\left(F_{n}\right)\right)=\sum_{i=1}^{r}\left(n_{v}\left(v v^{i}\right) n_{v^{i}}\left(v v^{i}\right)\right)+\sum_{i=1}^{n}\left(n_{v}\left(v v_{i}\right) n_{v_{i}}\left(v v_{i}\right)\right)+ \\
& \sum_{i=1}^{n-1}\left(n_{v_{i}}\left(v_{i} v_{i+1}\right) n_{v_{i+1}}\left(v_{i} v_{i+1}\right)\right)+\sum_{i=1}^{n} \sum_{j=1}^{r}\left(n_{v_{i}}\left(v_{i} v_{i}^{j}\right) n_{v_{i}^{j}}\left(v_{i} v_{i}^{j}\right)\right) \\
& =r(r+n(r+1))+\left(2(n-1)(r+1)^{2}+(n-2)^{2}(r+1)^{2}\right)+ \\
& 4(r+1)^{2}+4(n-3)(r+1)^{2}+n r(r+n(r+1)) \\
& =r^{2}\left(2 n^{2}+4 n-5\right)+r\left(3 n^{2}+5 n-12\right)+\left(n^{2}+2 n-6\right) .
\end{aligned}
$$

Corollary 5. $S z\left(F_{n}\right)=n^{2}+2 n-6$.

\section{Theorem 6.}

$$
S z\left(I_{r}\left(W_{n}\right)\right)=r^{2}\left(2 n^{2}+4 n+1\right)+r\left(3 n^{2}+5 n\right)+\left(n^{2}+2 n\right) .
$$

Proof. In view of the definition of Szeged index, we infer

$$
\begin{aligned}
& \operatorname{Sz}\left(I_{r}\left(W_{n}\right)\right)=\sum_{i=1}^{r}\left(n_{v}\left(v v^{i}\right) n_{v^{i}}\left(v v^{i}\right)\right)+\sum_{i=1}^{n}\left(n_{v}\left(v v_{i}\right) n_{v_{i}}\left(v v_{i}\right)\right)+ \\
& \sum_{i=1}^{n}\left(n_{v_{i}}\left(v_{i} v_{i+1}\right) n_{v_{i+1}}\left(v_{i} v_{i+1}\right)\right)+\sum_{i=1}^{n} \sum_{j=1}^{r}\left(n_{v_{i}}\left(v_{i} v_{i}^{j}\right) n_{v_{i}^{j}}\left(v_{i} v_{i}^{j}\right)\right) \\
& =r(r+n(r+1))+n(n-2)(r+1)^{2}+4 n(1+r)^{2}+ \\
& n r(r+n(r+1)) \\
& =r^{2}\left(2 n^{2}+4 n+1\right)+r\left(3 n^{2}+5 n\right)+\left(n^{2}+2 n\right) .
\end{aligned}
$$

Corollary 6. $S z\left(W_{n}\right)=n^{2}+2 n$.

Theorem 7.

$$
\begin{aligned}
& S z\left(I_{r}\left(\tilde{F}_{n}\right)\right)= \\
& r^{2}\left(22 n^{2}-43 n+28\right)+r\left(40 n^{2}-88 n+56\right)+\left(18 n^{2}-43 n+28\right) .
\end{aligned}
$$

Proof. By virtue of the definition of Szeged index, we yield

$$
\begin{aligned}
& S z\left(I_{r}\left(\tilde{F}_{n}\right)\right)=\sum_{i=1}^{r}\left(n_{v}\left(v v^{i}\right) n_{v^{i}}\left(v v^{i}\right)\right)+\sum_{i=1}^{n}\left(n_{v}\left(v v_{i}\right) n_{v_{i}}\left(v v_{i}\right)\right)+ \\
& \sum_{i=1}^{n} \sum_{j=1}^{r}\left(n_{v_{i}}\left(v_{i} v_{i}^{j}\right) n_{v_{i}^{j}}\left(v_{i} v_{i}^{j}\right)\right) \\
& \sum_{i=1}^{n-1}\left(n_{v_{i}}\left(v_{i} v_{i, i+1}\right) n_{v_{i, i+1}}\left(v_{i} v_{i, i+1}\right)\right)+ \\
& \sum_{i=1}^{n-1}\left(n_{v_{i, i+1}}\left(v_{i, i+1} v_{i+1}\right) n_{v_{i+1}}\left(v_{i, i+1} v_{i+1}\right)\right) \\
& \sum_{i=1}^{n-1} \sum_{j=1}^{r}\left(n_{v_{i, i+1}}\left(v_{i, i+1} v_{i, i+1}^{j}\right) n_{v_{i, i+1}^{j}}\left(v_{i, i+1} v_{i, i+1}^{j}\right)\right) \\
= & r(r+(r+1)(2 n-1))+8(n-1)(r+1)^{2}+ \\
& 3(n-2)(2 n-3)(r+1)^{2}+{ }_{+} n r(2 n(r+1)-1)+
\end{aligned}
$$

$$
\begin{aligned}
& 3(n-1)(2 n-3)(r+1)^{2}+3(n-1)(2 n-3)(r+1)^{2}+ \\
&(n-1) r(2 n(r+1)-1) \\
&= \\
& r^{2}\left(22 n^{2}-43 n+28\right)+r\left(40 n^{2}-88 n+56\right)+\left(18 n^{2}-43 n+28\right) .
\end{aligned}
$$

Corollary 7. $\operatorname{Sz}\left(\tilde{F}_{n}\right)=18 n^{2}-43 n+28$.

\section{Theorem 8.}

$S z\left(I_{r}\left(\tilde{W}_{n}\right)\right)=r^{2}\left(22 n^{2}-14 n+1\right)+r\left(40 n^{2}-34 n\right)+\left(18 n^{2}-18 n\right)$.

Proof. In view of the definition of Szeged index, we deduce

$$
\begin{aligned}
& P I_{v}\left(I_{r}\left(\tilde{W}_{n}\right)\right)=\sum_{i=1}^{r}\left(n_{v}\left(v v^{i}\right) n_{v^{i}}\left(v v^{i}\right)\right)+\sum_{i=1}^{n}\left(n_{v}\left(v v_{i}\right) n_{v_{i}}\left(v v_{i}\right)\right)+ \\
& \sum_{i=1}^{n} \sum_{j=1}^{r}\left(n_{v_{i}}\left(v_{i} v_{i}^{j}\right) n_{v_{i}^{j}}\left(v_{i} v_{i}^{j}\right)\right)
\end{aligned}
$$

$$
\begin{aligned}
& \sum_{i=1}^{n}\left(n_{v_{i}}\left(v_{i} v_{i, i+1}\right) n_{v_{i, i+1}}\left(v_{i} v_{i, i+1}\right)\right)+ \\
& \sum_{i=1}^{n}\left(n_{v_{i, i+1}}\left(v_{i, i+1} v_{i+1}\right) n_{v_{i+1}}\left(v_{i, i+1} v_{i+1}\right)\right) \\
& \sum_{i=1}^{n} \sum_{j=1}^{r}\left(n_{v_{i, i+1}}\left(v_{i, i+1} v_{i, i+1}^{j}\right) n_{v_{i, i+1}^{j}}\left(v_{i, i+1} v_{i, i+1}^{j}\right)\right) \\
= & r(r+2 n(r+1))+3 n(2 n-2)(r+1)^{2}+{ }^{2} n r((2 n+1)(r+1)-1)+ \\
& 3 n(2 n-2)(r+1)^{2}
\end{aligned}
$$$$
+3 n(2 n-2)(r+1)^{2}+n r((2 n+1)(r+1)-1)
$$$$
=r^{2}\left(22 n^{2}-14 n+1\right)+r\left(40 n^{2}-34 n\right)+\left(18 n^{2}-18 n\right)
$$

Corollary 8. $S z\left(\tilde{W}_{n}\right)=18 n^{2}-18 n$.

\section{CONFLICT OF INTEREST}

The authors confirm that this article content has no conflict of interest.

\section{ACKNOWLEDGEMENTS}

First, we thank the reviewers for their constructive comments in improving the quality of this paper. This work was supported in part by the National Natural Science Foundation of China (61262071), and the Key Science and Technology Research Project of Education Ministry (210210). We also would like to thank the anonymous referees for providing us with constructive comments and suggestions.

\section{REFERENCES}

[1] Yan L, Li Y, Gao W, Li J. On the extremal hyper-wiener index of graphs, J Chem Pharmaceut Res 2014; 6(3): 477-81.

[2] Yan L, Li Y, Gao W, Li J. PI index for some special graphs. J Chem Pharmaceut Res 2013; 5(11): 260-64.

[3] Gao W, Shi L. Wiener index of gear fan graph and gear wheel graph. Asian J. Chem 2014; 26(11): 3397-400,. 
[4] Khalifeh MH, Yousefi-Azari H, Ashrafi AR. Vertex and edge PI indices of cartesian product graphs. Discrete Appl Math 2008; 156: 1780-89.

[5] Ashrafi AR, Ghorbani M, Jalali M. The vertex PI and Szeged indices of an infinite family of fullerenes. J Theor Comput Chem 2008; 7(2): 221-31.

[6] Khalifeh MH, Yousefi-Azari H, Ashrafi AR. A matrix method for computing Szeged and vertex PI indices of join and composition of graphs. Linear Alg Appl 2008; 429(11-12): 2702-09.

[7] Yousefi-Azari H, Ashrafi AR, Khalifeh MH. Computing vertex-PI index of single and multi-walled nanotubes. Digest J Nanomat Biostruct. 2008; 3(4): 315-318.

[8] Mansour T, Schork M. The vertex PI index and Szeged index of bridge graphs. Discrete Appl Math 2009; 157(7): 1600-06.
[9] Nadjafi-Arani MJ, Fath-Tabar GH, Ashrafi AR. Extremal graphs with respect to the vertex PI index. Appl Math Lett 2009; 22: 183840.

[10] Das K, Gutman I. Bound for vertex PI index in terms of simple graph parameters, Filomat 2013; 27(8): 1583-87.

[11] Li X, Yang X, Wang G, Hu R. The vertex PI and Szeged indices of chain graphs, MATCH Commun. Math Comput Chem 2012; 68: 349-56.

[12] Bahrami A, Yazdani J. Vertex PI index of V-phenylenic nanotubes and nanotori. Digest J. Nanomat. Biostruct 2009; 4(1): 141-144.

[13] Yousefi-Azari H, Manoochehrian B, Ashrafi AR. Szeged index of some nanotubes. Curr Appl Phy 2008; 8(6): 713-715.

Received: September 22, 2014

(C) Yan et al.; Licensee Bentham Open.

This is an open access article licensed under the terms of the Creative Commons Attribution Non-Commercial License (http://creativecommons.org/licenses/ by-nc/3.0/) which permits unrestricted, non-commercial use, distribution and reproduction in any medium, provided the work is properly cited. 